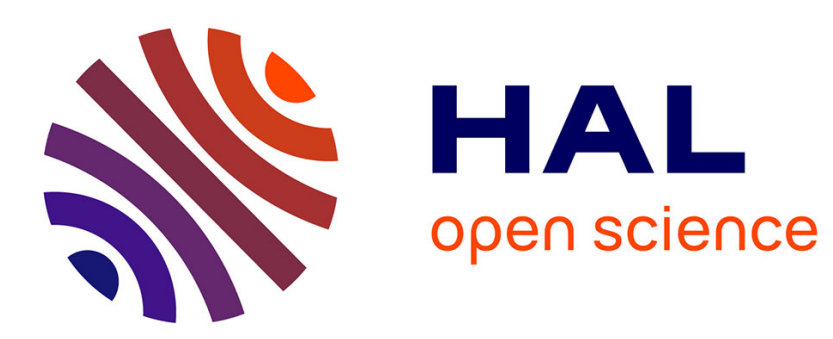

\title{
Grid technology for biomedical applications
}

\author{
Vincent Breton, Christophe Blanchet, L. Maigne, Johan Montagnat
}

\section{To cite this version:}

Vincent Breton, Christophe Blanchet, L. Maigne, Johan Montagnat. Grid technology for biomedical applications. VECPAR 2004 Conference, Jun 2004, Valencia, Spain. pp.204-218, 10.1007/b106965 . in2p3-00171787

\section{HAL Id: in2p3-00171787 https://hal.in2p3.fr/in2p3-00171787}

Submitted on 13 Sep 2007

HAL is a multi-disciplinary open access archive for the deposit and dissemination of scientific research documents, whether they are published or not. The documents may come from teaching and research institutions in France or abroad, or from public or private research centers.
L'archive ouverte pluridisciplinaire HAL, est destinée au dépôt et à la diffusion de documents scientifiques de niveau recherche, publiés ou non, émanant des établissements d'enseignement et de recherche français ou étrangers, des laboratoires publics ou privés. 


\title{
Grid Technology for Biomedical Applications
}

\author{
Vincent Breton $^{1}$, Christophe Blanchet ${ }^{2}$ Lydia Maigne $^{1}$ and Johan Montagnat ${ }^{3}$ \\ ${ }^{1}$ LPC, CNRS-IN2P3 / Université Blaise Pascal, Campus des Cézeaux, 63177 Aubière Ce- \\ dex, France \\ (Breton, Maigne) @clermont.in2p3.fr \\ 2 IBCP, CNRS, 7, passage du Vercors, 69367 Lyon CEDEX 07, France \\ Christophe.Blanchet@ibcp.fr \\ ${ }^{2}$ CREATIS, CNRS UMR5515- INSERM U630, INSA, 20 Ave. A. Einstein, Villeurbanne, \\ France \\ johan@i3s.unice.fr
}

\begin{abstract}
The deployment of biomedical applications in a grid environment has started about three years ago in several European projects and national initiatives. These applications have demonstrated that the grid paradigm was relevant to the needs of the biomedical community. They have also highlighted that this community had very specific requirements on middleware and needed further structuring in large collaborations in order to participate to the deployment of grid infrastructures in the coming years. In this paper, we propose several areas where grid technology can today improve research and healthcare. A crucial issue is to maximize the cross fertilization among projects in the perspective of an environment where data of medical interest can be stored and made easily available to the different actors of healthcare, the physicians, the healthcare centres and administrations, and of course the citizens.
\end{abstract}

\section{Introduction}

Last summer, about 10000 elderly people died in one European country because of unusually long and severe hot weather. For two weeks, the overall increase of mortality rate in hospitals and healthcare centres remained unnoticed. To better handle this kind of situation, a strategy is to set up a monitoring service recording daily on a cen- 
tral repository the number of casualties in each healthcare centre. With the present telemedicine tools, such a monitoring service requires an operator in each healthcare centre to submit the information to the central repository and an operator to validate the information provided. In case of emergency, for instance if the monitoring service identifies an abnormal increase of the mortality rate, experts have to be called to analyze the information available at the central repository. If they want additional information, they need to require it from the operators in each healthcare centre. This extra request may introduce major delays and extra work on health professionals who are already overworked.

With the onset of grid technology, such a monitoring service would require much less manpower. Indeed, grid technology delivers today access in a secure way to data stored on distant grid nodes. Instead of having one operator in each centre in charge of transmitting information daily to the central repository, the information on the number of casualties is stored locally on a database which is accessible by the central repository. In case of emergency, the experts can access further to the healthcare centre database to inquire about the patient medical files. In this scenario, patient medical files stay in healthcare centres and the central monitoring service picks up only what is needed for its task.

\section{Vision for a grid for health}

The example used to introduce this paper illustrates the potential impact of grid technology for health. The grid technology is identified as one of the key technologies to enable the European research Area. Its impact is expected to reach much beyond eScience to eBusiness, eGouvernment, ... and eHealth. However, a major challenge is to take the technology out of the laboratory to the citizen. A HealthGrid (figure 1) is an environment where data of medical interest can be stored and made easily available to the different actors of healthcare, the physicians, the healthcare centres and administrations, and of course the citizens. Such an environment has to offer all guarantees in terms of security, respect of ethics and regulations. Moreover, the association of post-genomics and medical data on such an environment opens the perspective of individualized healthcare.

While considering the deployment of life sciences applications, most present grid projects do not address the specificities of an e-infrastructure for health, for instance the deployment of grid nodes in clinical centres and in healthcare administrations, the connection of individual physicians to the grid and the strict regulations ruling the access to personal data,.... 

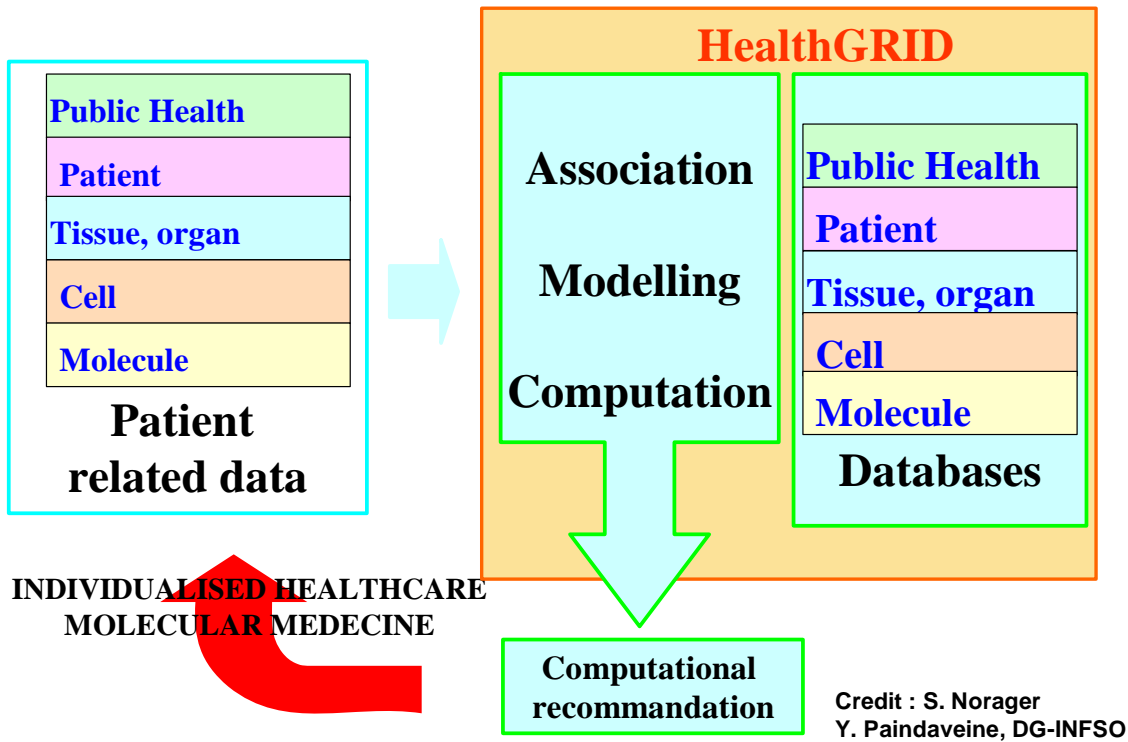

Fig. 1. Pictorial representation of the Healthgrid concept

Technology to address these requirements in a grid environment is under development and a pioneering work is under way in the application of Grid technologies to the health area.

In the last couple of years, several grid projects have been funded on health related issues at national and European levels. These projects have a limited lifetime, from 3 to 5 years, and a crucial issue is to maximize their cross fertilization. Indeed, the Healthgrid is a long term vision that needs to build on the contribution of all projects. The Healthgrid initiative, represented by the Healthgrid association (http://www.healthgrid.org), was initiated to bring the necessary long term continuity. Its goal is to collaborate with projects on the following activities:

- Identification of potential business models for medical Grid applications;

- Feedback to the Grid-development community on the requirements of the pilot applications deployed by the European projects;

- Dialogue with clinicians and people involved in medical research and Grid development to determine potential pilots;

- Interaction with clinicians and researchers to gain feedback from the pilots;

- Interaction with all relevant parties concerning legal and ethical issues identified by the pilots;

- Dissemination to the wider biomedical community on the outcome of the pilots; 
- Interaction and exchange of results with similar groups worldwide;

- Definition of potential new applications in conjunction with the end user communities.

\section{Requirements}

To deploy the monitoring service described in introduction to this article, patient medical data have to be stored in a local database in each healthcare centre. These databases have to be federated and must be interoperable. Secure access to data is mandatory: only views of local data should be available to external services and patient data should be anonymized when nominative information is not needed.

These requirements have to be fed back to the community developing middleware services. The Healthgrid initiative is actively involved in the definition of requirements relevant to the usage of grids for health. As an example of a first list of requirements, the one produced by DataGrid can be given.

\subsection{Data related requirements}

- Provide access to biology and medical image data from various existing databases

- Support and improve existing databases import/export facilities

- Provide transparent access to data from the user point of view, without knowledge of their actual location

- Update databases while applications are still running on their own data versions

\subsection{Security related requirements}

- Grant anonymous and private login for access to public and private databases

- Guarantee the privacy of medical information

- Provide an encryption of sensitive message

- Fulfill all legal requirements in terms of data encryption and protection of patient privacy

- Enforce security policies without the need to modify applications

\subsection{Administration requirements}

- Provide "Virtual Grids", ie the ability to define subgrids with a restricted access to data and computing power 
- Provide "Virtual Grids" with customizable login policies

\subsection{Network requirements}

- Provide batch computing on huge dataset

- Provide fast processing applications which transfer small set of images between different sites : storage site, processing site and physician site

- Provide interactive access to small data sets, like image slices and model geometry

\subsection{Job related requirements}

- Allow the user to run jobs "transparently", without the knowledge of the underlying scheduling mechanism and resource availability

- Manage jobs priorities

- Deal with critical jobs : if no resource are available, jobs with lowest priority will be interrupted to allow execution of critical jobs

- Permit to chain batch jobs into pipelines

- Provide a fault-tolerant infrastructure with fault detection, logging and recovery

- Notify an user when a job fails for a Grid independent reason

- Provide an interactive mode for some applications

- Support for parallel jobs

- Provide a message passing interface inside a local farm and also at the Grid level

- Offer Job monitoring and control, i.e. query job status, cancel queuing or running job

- Provide logs to understand system failures and intruders detection.

\section{DataGrid}

The deployment of biomedical applications in a grid environment has started about three years ago in several European projects and national initiatives. These applications have demonstrated that the grid paradigm was relevant to the needs of the biomedical community.

The European DataGrid (EDG) project [1], which started three years ago, successfully concluded on 31 March 2004. It aimed at taking a major step towards making the concept of a world-wide computing Grid a reality. The goal of EDG was to build a test computing infrastructure capable of providing shared data and computing resources across the European scientific community. The budget for the project was around 10 million euros and 21 partner institutes and organizations across Europe were involved. 
After a massive development effort involving seven major software releases over three years, the final version of EDG software is already in use in three major scientific fields: High Energy Physics, Biomedical applications and Earth Observations. At peak performance, the EDG test bed shared more than 1000 processors and more than 15 Terabytes of disk space spread in 25 sites across Europe, Russia and Taïwan. The software is exploited by several bio-medical applications in the area of bioinformatics and biomedical simulation.

\subsection{Monte-Carlo simulation for nuclear medicine and radio/brachytherapy}

The principle of Monte-Carlo simulations is to reproduce radiation transport knowing the probability distributions governing each interaction of particles in the patient body and in the different equipments needed in nuclear medicine and brachy/radiotherapy: gamma-camera and PET for nuclear medicine, accelerator head for radiotherapy and internal radiation applicators for brachytherapy. Accuracy is therefore only limited by the number of particles generated. As a consequence, Monte-Carlo simulations are increasingly used in nuclear medicine to generate simulated PET and SPECT images to assess the performances of reconstruction algorithms in terms of resolution, sensitivity and quantification and to design innovative detectors. In external beam radiotherapy, Monte-Carlo simulations are needed for accelerator head modelling and computation of beam phase space [2]. Accelerator beam modelling is especially critical to reach high accuracy in gradient and shielded regions for Intensity Modulated Radiation Therapy. Measurement of dose deposit by applicators in brachytherapy is often difficult experimentally because of high gradient. In the case of electron sources, Monte-Carlo simulation is especially relevant provided electron transport is properly described.

All in all, the major limiting factor for the clinical implementation of Monte-Carlo dose calculations methods is the large computing time requested to reach the desired accuracy. Most of the commercial systems, named TPS (Treatment Planning Systems), for clinical routine use an analytic calculation to determine dose distributions and so, errors near heterogeneities in the patient can reach 10 to $20 \%$. Such codes are very fast comparing to Monte Carlo simulations: the TPS computation time for an ocular brachytherapy treatment is lower than on minute, thus allowing its usage in clinical practice, while a Monte Carlo framework could take 2 hours.

To evaluate the impact of parallel and distributed Monte Carlo simulations, a radiotherapy treatment planning was performed on the EDG Testbed, from pre-processing and registration of medical images on the Storage Elements (SEs) of the grid to the parallel computation of Monte Carlo simulations GATE (Geant4 Application for Tomographic Emission [3].

The application framework is depicted in figure 3. Sets of 40 DICOM slices or so, 5122 pixels each, acquired by CT scanners are concatenated and stored in a 3D image format. Such image files can reach until $20 \mathrm{MB}$ for our application. To solve privacy issues, DICOM headers are wiped out in this process. 


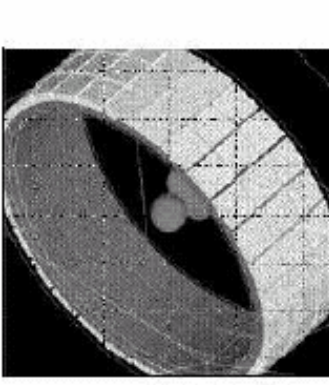

a)

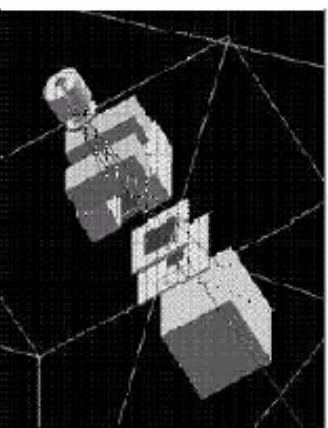

b)

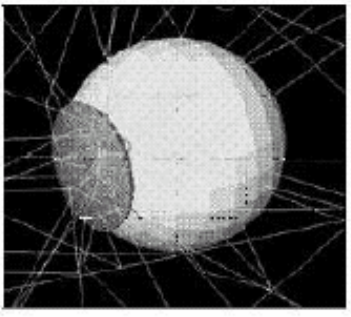

c)

Fig. 2. GATE Monte Carlo simulations: a) PET simulation; b) Radiotherapy simulation; c) Ocular brachytherapy simulation

The 3D image files are then registered and replicated on the sites of the EDG testbed where GATE is installed in order to compute simulations (5 sites to date). During the computation of the GATE simulation, the images are read by GATE and interpreted in order to produce a 3D array of voxels whose value is describing a body tissue. A relational database is used to link the GUID of image files with metadata extracted from the DICOM slices on the patient and additional medical information. The EDG Spitfire software is used to provide access to the relational databases.

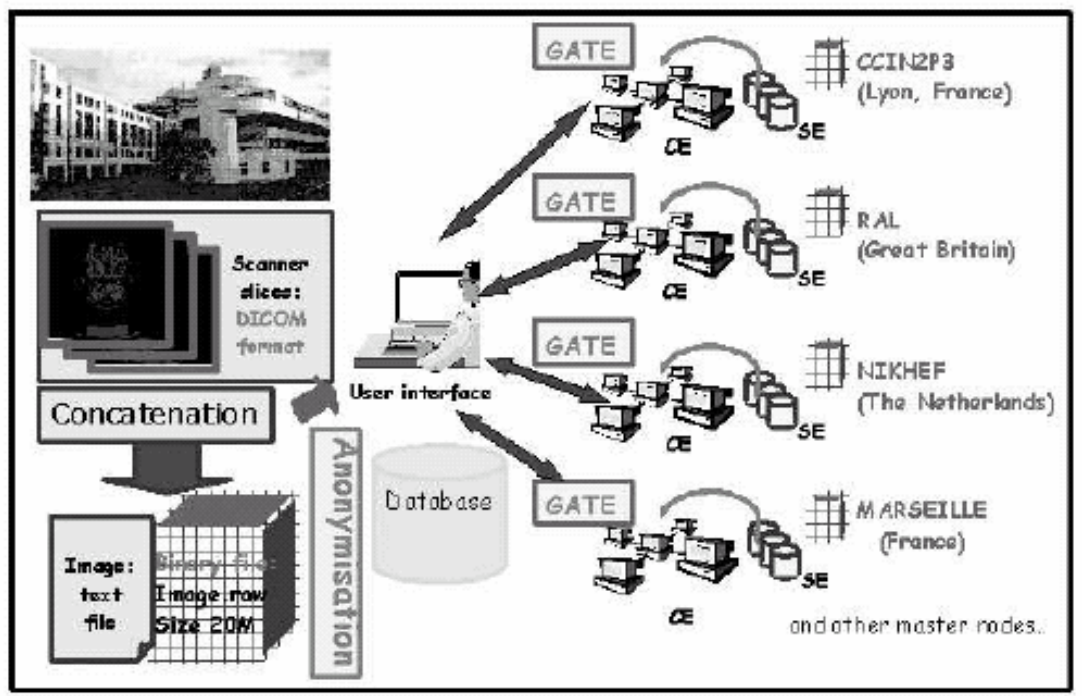

Fig. 3. Submission of GATE jobs on the DataGrid testbed 
Every Monte Carlo simulation is based on the generation of pseudorandom numbers using a Random Numbers Generator (RNG). An obvious way to parallelize the calculations on multiple processors is to partition a sequence of random numbers generated by the RNG into suitable independent sub-sequences.

To perform this step, the choice has been done to use the Sequence Splitting Method [4],[5]. For each sub-sequences, we save in a file (some KBs) the current status of the random engine. Each simulation is then launched on the grid with the

status file. All the other files necessary to run Gate on the grid are automatically created: the script describing the environment of computation, the macros GATE describing the simulations, the status files of the RNG and the job description files.

In order to show the advantage for the GATE simulations to partition the calculation on multiple processors, the simulations were split and executed in parallel on several grid nodes. Table 1 illustrates the computing time in minutes of a GATE simulation running on a single $\mathrm{P} 4$ processor at $1.5 \mathrm{GHz}$ locally and the same simulation splitting by $10,20,50$ and 100 jobs on multiple processors [6].

Table 1. Sequential versus grid computation time using 10 to 100 nodes

\begin{tabular}{lccccc}
\hline Number of jobs submitted & 10 & 20 & 50 & 100 & Local \\
\hline Total computing time in minutes & 31 & 20,5 & 31 & 38 & 159
\end{tabular}

The results show a significant improvement in computation time although the computing time using Monte Carlo calculations should stay comparable to what it is currently with analytical calculations for clinical practice. The next challenge is to provide the necessary quality of service requested by the medical user to compute his simulation on the grid.

\subsection{Bioinformatics grid-enabled portals}

One of the current major challenges in the bioinformatics field is to derive valuable information from ongoing complete genome sequencing projects (currently 1087 genome projects with 182 published ones), which provide the bioinformatics community with a large number of sequences. The analysis of such huge sets of genomic data requires big storage and computing capacities, accessible through user-friendly interfaces such as web portals. Today, the genomic and post-genomic web portals available rely on their local cpu and storage resources. 


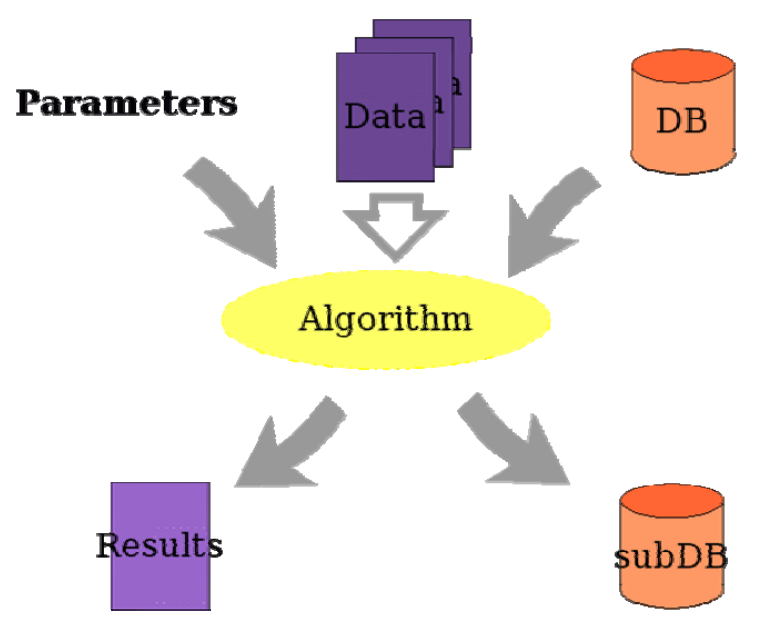

Fig. 4: Bioinformatics algorithm schema

Grid computing may be a viable solution to go beyond these limitations and to bring computing resources suitable to the genomic research field.

A solution explored in the European DataGrid project was to interface the NPS@ web

site [ref.] dedicated to protein structure analysis to the DataGrid infrastructure.

The bioinformatics sequence algorithms used on NPS@ web portal are of different types depending on the data analyses they aim to compute: sequence homology and similarity searching (e.g. BLAST, FASTA), patterns and signatures searching (e.g. PattInProt), multiple alignment of proteins (e.g. ClustalW), secondary structure prediction and so on. The "gridification" of web portals for genomics has to deal with these different algorithms and their associated models concerning their storage and CPU resources consumption (Figure 4). The bioinformatics algorithms for protein analysis can be classified into 4 categories on the criteria of CPU resources and input/output data requirements (Table 2).

According to its class, an algorithm is sent to the grid in a batch mode by distributing the algorithm and creating subsets of the input data or in a MPI-like (message passing interface) execution context by sending sub processes to different nodes of the grid (although this mode isn’t currently stable enough to be used in DataGrid).

\begin{tabular}{|c|c|c|c|}
\hline & & \multicolumn{2}{|l|}{ I/O DATA } \\
\hline & & Small & Large \\
\hline $\begin{array}{l}\bar{\Xi} \\
\text { ई }\end{array}$ & 窇 & $\begin{array}{l}\text { Protein secondary structure pre- } \\
\text { diction (GOR, DPM, } \\
\text { Simpa96...). } \\
\text { Physicochemical profiles... }\end{array}$ & $\begin{array}{l}\text { BLAST } \\
\text { ProScan (protein pattern) } \\
\ldots\end{array}$ \\
\hline $\begin{array}{l}0 \\
0 \\
0 \\
0 \\
0\end{array}$ & : & $\begin{array}{l}\text { Multiple alignement with } \\
\text { CLUSTAL W or Multalin } \\
\ldots\end{array}$ & $\begin{array}{l}\text { FASTA, SSEARCH } \\
\text { PattInProt (protein pattern) } \\
\text { protein secondary structure prediction } \\
\text { (SOPMA, PHD,...) } \\
\text { CLUSTAL W (on complete ge- } \\
\text { nnmes) }\end{array}$ \\
\hline
\end{tabular}


Table 2. Classification of the bioinformatics algorithms used in GPS@ according to their data and CPU requirements.

In fact, the major problem with a grid computing infrastructure is the distribution of the data and their synchronization according to the current release. Moving on the grid a databank which size varies from tens of megabytes (e.g. SwissProt) to gigabytes (e.g. EMBL), requires a significant fraction of the network bandwith and therefore increases the execution time. One simple solution can be to split databanks into subsets sent in parallel to several grid nodes, in order to run the same query on each

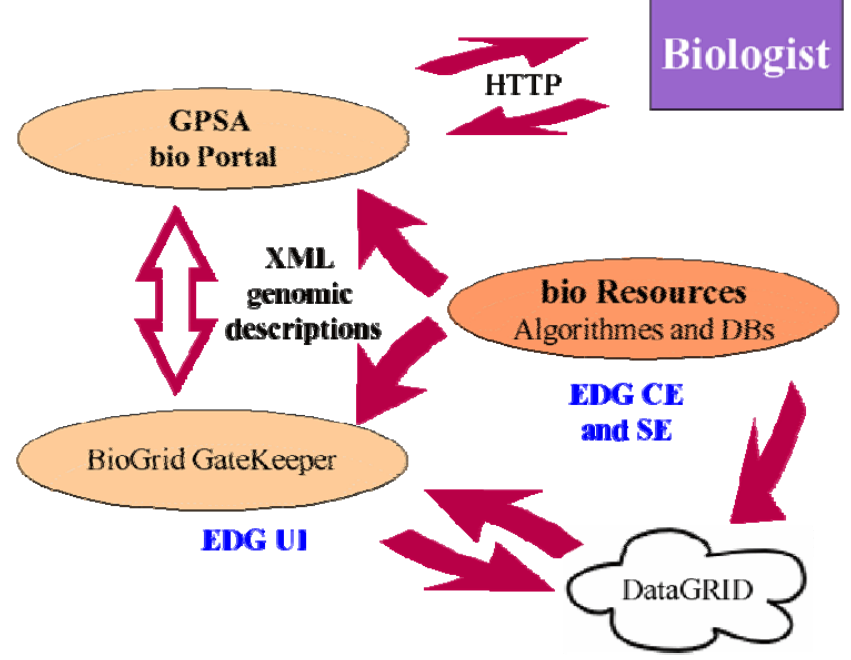

Fig. 5: Bioinformatic job processing on GPS@ web portal

subset. Such approach requires a synchronization of all the output files at the end. A more efficient solution would be to update the databanks on several referenced nodes with the help of the Replica Manager provided by the DataGrid middleware and to launch the selected algorithms on these nodes. To summarize, the algorithms working on short dataset are sent at runtime with the data through the grid sandbox while the ones analyzing large datasets are executed on the grid nodes where the related databanks have been transported earlier by the DataGrid replica manager service.

GPS@ - Grid Protein Sequence Analysis. Depending on the algorithms, DataGrid submission process is different. Some of the algorithms on NPS@ portal have been adapted in agreement to the DataGrid context, and will be made available to the biologist end-user in a real production web portal in the future (they can be tested on the URL http://gpsa.ibcp.fr). GPS@ offers the same bioinformatics queries as NPS@, with the addition of job submission on DataGrid resources accessible through a drag-and-drop mechanism and simply pressing the "submit" button. All the DataGrid job management is encapsulated into the GPS@ backoffice: scheduling and 
status of the submitted jobs (Fig. 5). And finally the result of the biological grid jobs are displayed into a new web page, ready for further analysis or for download

\subsection{Humanitarian medical development}

The training of local clinicians is the best way to raise the standard of medical knowledge in developing countries. This requires transferring skills, techniques and resources. Grid technologies open new perspectives for preparation and follow-up of medical missions in developing countries as well as support to local medical centres in terms of teleconsulting, telediagnosis, patient follow-up and e-learning. To meet requirements of a development project of the French NPO Chain of Hope in China, a first protocol was established for describing the patient pathologies and their pre- and post-surgery states through a web interface in a language-independent way. This protocol was evaluated by French and Chinese clinicians during medical missions in the fall 2003 [7]. The first sets of medical patients recorded in the databases will be used to evaluate grid implementation of services and to deploy a grid-based federation of databases. Such a federation of databases keeps medical data distributed in the hospitals behind firewalls. Views of the data will be granted according to individual access rights through secured networks.

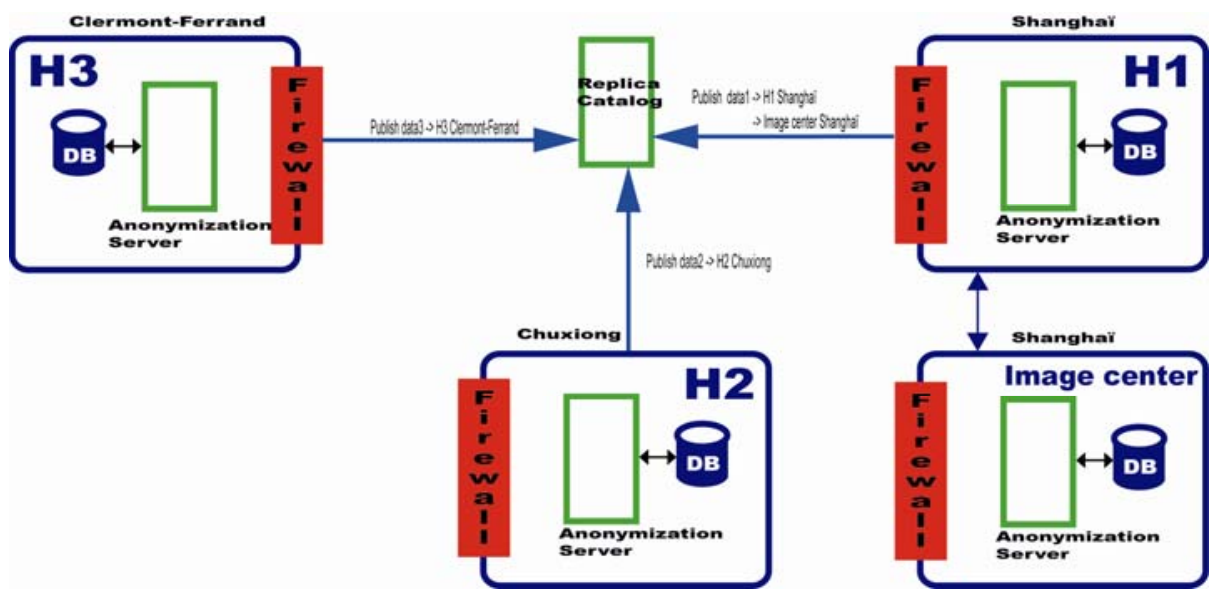

Fig. 4. Schematic representation of data storage architecture and information flow between three hospitals.

\subsection{Medical image storage, retrieval and processing}

Medical images represent tremendous amounts of data produced in healthcare centers each day. Picture Archiving and Communication Systems (PACS) are proposed by 
imager manufacturers to help hospitals archiving and managing their images. In addition to PACS, Radiological Information Systems (RIS) are deploy to store medical records of patients. PACS and RIS are developped with an clinical objective of usability. However, they usually do not address the problems of large scale (cross-site) medical data exchange as most of them are proprietary manufacturers solution that do not rely on any existing standard for interoperability. Moreoever, they are usually weakly addressing the security problems arising in wide scale grid computing since they are usually bounded to each health center. Finally, they do not consider the increasing need for automated data analysis and they do not offer any interface to external computing resources such as a grid.

Grids are a logical extension of regional PACS and RIS. However, many technical issues as well as an adaptation of the medical world to Information Technology tools delay the wide applicability of grid technologies in this field. In the European DataGrid project and the French National MEDIGRID project [5], we have been working on medical data management and grid processing on medical data [6].

\subsubsection{Medical data management}

In order to grid enable medical image processing application, it is necessary to interface the hospitals medical information system with the grid. Figure 4 illustrates the Distribued Medical Data Manager (DM2) developped in the MEDIGRID project. The DM2 is an interface between the grid middleware and the medical server. On the hospital side, it interfaces to Digital Image and COmmunication in Medicine (DICOM) servers. DICOM is the most widely accepted standard for medical images storage and communication. Most recent medical imagers are DICOM compliant: they produce images that they can locally archive or transfer to a DICOM server. The DM2 can be conneted to one central or several distributed DICOM servers available in the hospital. On the grid side, the DM2 provides a grid storage interface. Each time a medical image is recored on the DICOM server, the DM2 registers a new file entry on the grid data management system. It also contains a database storing metadata (the medical record) associated to images. From the grid side, images recorded are accessible to authorized users as any file regularly registered. The image can be accessed by a grid node through the DM2 interface which translates the grid incoming request into a DICOM request and returns the desired file. However, the DM2 provide additional services such as automatic data anonymization and encryption to prevent critical data from being accessible by non accredited users. See [8] for details. 


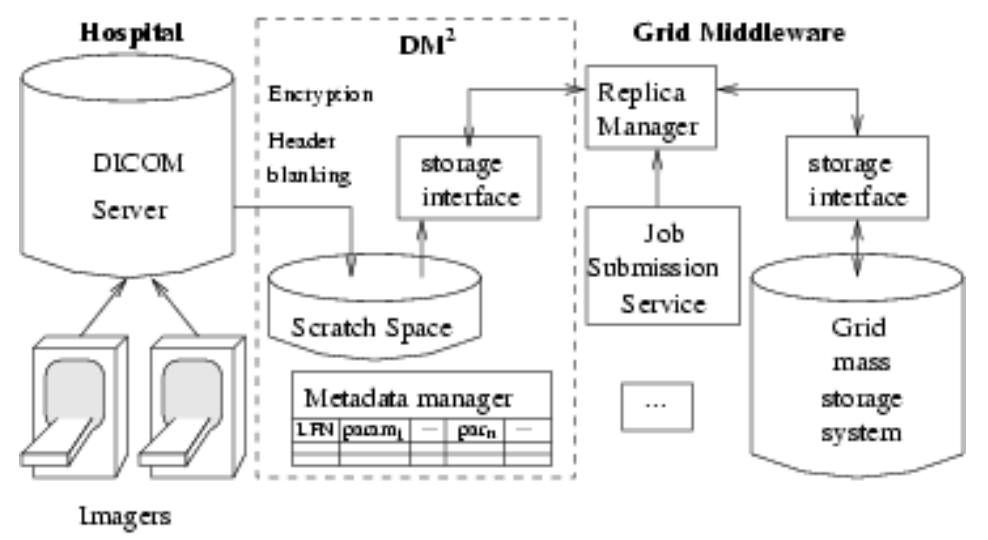

Fig. 5. $\mathrm{DM}^{2}$ : A Distributed Medical Data Manager

\subsubsection{Medical data processing}

Through an interface such as the DM2 a grid can offer an access to large medical image and metadata databases spread over different sites. This is usefull for many data intensive medical image processing applications for which large datasets are needed. Moreover, grids are well suited to handle computation resulting from exploring full databases. For instance, epidemiology is a field requiring to collect statistics over large population of patients. For rare pathologies, this can only be achieved if data providing from a large number of sites can be assembled. Building digital atlases also requires to assemble large image sets.

An example of a grid image content-based retrieval application is described in detail in [7] and depicted in figure 5. The typical scenario is a medical doctor diagnosing a medical image of one of his/her patient that has been previously registered $(1,2)$. To confirm his/her diagnosis, he/she would like to confront it to similar known medical cases. The application allows the physician to search for recorded images similar to the source image he is interested in. Target candidates are first selected out of the database by querrying image metadata (3). Several similarity criterion may then be used to compare each image from the database to the source image. The similarity compuation algorithms return a score. Images of the database can be ranked according to this score and the physician can download highest score images corresponding to most similar cases. This application requires one similarity job to be started for each candidate image to be compared in the database (4). These computations are distributed over available grid nodes to speed up the search $(5,6)$. Given the absence of dependencies between the computations, the computing time is therefore divided by the number of processor available, neglecting the grid overhead (network transmission of data and scheduling overhead). This application has been successfully deployed on the EDG testbed, processing up to hundreds of images (tens of images are processed in parallel). 


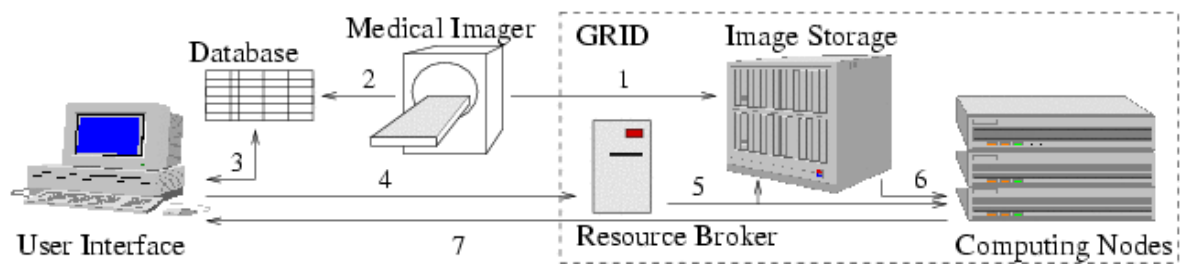

Fig. 6. Application synopsis

\section{An emerging project: a grid to address a rare disease}

Another area where grid technology offers promising perspectives for health is drug discovery. This chapter presents the potential interest of a grid dedicated to Research and development on a rare disease.

\subsection{The crisis of neglected diseases}

There is presently a crisis in research and development for drugs for neglected diseases. Infectious diseases kill 14 million people each year, more than ninety percent of whom are in the developing world. Access to treatment for these diseases is problematic because the medicines are unaffordable, some have become ineffective due to resistance, and others are not appropriately adapted to specific local conditions and constraints. Despite the enormous burden of disease, drug discovery and development targeted at infectious and parasitic diseases in poor countries has virtually ground to a standstill, so that these diseases are de facto neglected. Of the 1393 new drugs approved between 1975 and 1999, less than 1\% (6) was specifically for tropical diseases. Only a small percentage of global expenditure on health research and development, estimated at US\$50-60 billion annually, is devoted to the development of such medicines. At the same time, the efficiency of existing treatments has fallen, due mainly to emerging drug resistance.

The unavailability of appropriate drugs to treat neglected diseases is among other factors a result of the lack of ongoing R\&D into these diseases. While basic research often takes place in university or government labs, development is almost exclusively done by the pharmaceutical industry, and the most significant gap is in the translation of basic research through to drug development from the public to the private sector. Another critical point is the launching of clinical trials for promising candidate drugs. Producing more drugs for neglected diseases requires building a focussed, diseasespecific R\&D agenda including short-, mid- and long-term projects. It requires also a public-private partnership through collaborations that aims at improving access to drugs and stimulating discovery of easy-to-use, affordable, effective drugs. 


\subsection{The grid impact}

The grid should gather:

1. drug designers to identify new drugs

2. healthcare centres involved in clinical tests

3. healthcare centres collecting patent information

4. structures involved in distributing existing treatments (healthcare administrations, non profit organizations,...)

5. IT technology developers

6. Computing centres

7. Biomedical laboratories searching for vaccines, working on the genomes of the virus and/or the parasite and/or the parasite vector

The grid will be used as a tool for:

1. Search of new drug targets through post-genomics requiring data management and computing

2. massive docking to search for new drugs requiring high performance computing and data storage

3. handling of clinical tests and patent data requiring data storage and management

4. overseeing the distribution of the existing drugs requiring data storage and management

A grid dedicated to research and development on a given disease should provide the following services:

1. large computing resources for search for new targets and virtual docking

2. large resources for storage of post genomics and virtual docking data output

3. grid portal to access post genomics and virtual docking data

4. grid portal to access medical information (clinical tests, drug distribution,...)

5. a collaboration environment for the participating partners. No one entity can have an impact on all R\&D aspects involved in addressing one disease.

Such a project would build the core of a community pioneering the use of gridenabled medical applications. The choice of a neglected disease should help reducing the participants reluctance to share information. However, the issue of Intellectual Property must be addressed in thorough details.

\section{Conclusion}

The grid technology is identified as one of the key technologies to enable the European research Area. Its impact is expected to reach much beyond eScience to eBusiness, eGouvernment, ... and eHealth. However, a major challenge is to take the technology out of the laboratory to the citizen. A HealthGrid is an environment where data of medical interest can be stored and made easily available to the different actors of healthcare, the physicians, the healthcare centres and administrations, and of 
course the citizens. Such an environment has to offer all guarantees in terms of security, respect of ethics and regulations. Moreover, the association of post-genomics and medical data on such an environment opens the perspective of individualized healthcare.

The deployment of biomedical applications in a grid environment has started about three years ago in several European projects and national initiatives. These applications have demonstrated that the grid paradigm was relevant to the needs of the biomedical community.

In this paper, we have reported on our experience in the deployment of biomedical grid applications within the framework of the DataGrid project.

$\cdots$

\section{References}

1. Special issue of Journal of Grid Computing dedicated to DataGrid, to be published in 2004

2. ..., Issues limiting the clinical use of Monte Carlo dose calculation algorithms, Med. Phys. 30, vol. 12, (2003) 3206-3216

3. G. Santin, D. Strul, D. Lazaro, L. Simon, M. Krieguer, M. Vieira Martins, V. Breton and C. Morel. GATE, a Geant4-based simulation platform for PET and SPECT integrating movement and time management, IEEE Trans. Nucl. Sci. 50 (2003) 1516-1521

4. Traore, M. and Hill, D. (2001). The use of random number generation for stochastic distributed simulation: application to ecological modeling. In 13th European Simulation Symposium, Marseille, pages 555\{559, Marseille, France.

5. Coddington, P., editor (1996). Random Number Generators For Parallel Computers, Second Issue. NHSE Review.

6. Maigne, L., Hill, D., Breton, V., and et al. (2004). Parallelization of Monte Carlo simulations and submission to a Grid environment. Accepted for publication in Parallel Processing Letters.

7. V. J. Gonzales, S. Pomel, V. Breton, B. Clot, JL Gutknecht, B. Irthum, Y. Legré. Empowering humanitarian medical development using grid technology, submitted to proceedings of Healthgrid 2004 to be published in Methods of Information in Medecine

7. MEDIGRID project, French ministry for research ACI-GRID project, http://www.creatis.insa-lyon.fr/MEDIGRID/

8. J. Montagnat, V. Breton, I. E. Magnin, Using grid technologies to face medical image analysis challenges, Biogrid'03, proceedings of the IEEE CCGrid03, pp 588593, May 2003, Tokyo, Japan. 
9. J. Montagnat, H. Duque, J.M. Pierson, V. Breton, L. Brunie, I. E. Magnin, Medical Image Content-Based Queries using the Grid, HealthGrid'03, pp 138-147, January, 2003, Lyon, France

10. H. Duque, J. Montagnat, J.M. Pierson, L. Brunie, I. E. Magnin, DM2: A Distributed Medical Data Manager for Grids, Biogrid'03, proceedings of the IEEE CCGrid03, pp 606-611, May 2003, Tokyo, Japan. 